\title{
Use of Thermogravimetric Analysis for Moisture Determination in Difficult Lyophilized Biological Samples
}

\author{
Paul Matejtschuk1 ${ }^{1}$ Chinwe Duru1, Kiran Malik¹, Ernest Ezeajughi', Elaine Gray², \\ Sanj Raut ${ }^{2}$, Fatme Mawas ${ }^{3}$ \\ ${ }^{1}$ Standardization Science, National Institute for Biological Standards \& Control (NIBSC), Hertfordshire, UK \\ ${ }^{2}$ Biotherapeutics Division, National Institute for Biological Standards \& Control (NIBSC), Hertfordshire, UK \\ ${ }^{3}$ Bacteriology Division, National Institute for Biological Standards \& Control (NIBSC), Hertfordshire, UK \\ Email: paul.matejtschuk@nibsc.org
}

Received 21 January 2016; accepted 12 March 2016; published 15 March 2016

Copyright $@ 2016$ by authors and Scientific Research Publishing Inc.

This work is licensed under the Creative Commons Attribution-NonCommercial International License (CC BY-NC).

http://creativecommons.org/licenses/by-nc/4.0/

(c) (i) \&) Open Access

\begin{abstract}
Residual moisture is a key quality control parameter for lyophilized biologicals, as high moisture can correlate with poor stability. Coulometric Karl Fischer titration is the most widely used technology to determine residual water content; some chemicals are known to cause problems with Karl Fischer titration, but these chemicals do not usually occur in biologics. Three biological samples, of fibrinogen, heparin and Haemophilus influenza b polysaccharide, have caused particular issue in our hands by routine Karl Fischer analysis, illustrating different limitations with this method. The use of thermogravimetric analysis, with evolved gas mass spectrometric monitoring, is described here as a successful alternative for moisture analysis in these materials.
\end{abstract}

\section{Keywords}

Thermogravimetric Analysis, Freeze Dried, Moisture Determination, Karl Fischer Titration

\section{Introduction}

Lyophilization is a widely used technology for the stabilisation of biologicals, a group of pharmaceuticals which can be especially prone to degradation when stored in the liquid state [1]. A critical quality control parameter in these lyophilized materials is the residual water content [2], as high residual moisture has been correlated to poor stability [3]. A number of methods for moisture determination have been documented including gravime- 
tric methods, such as thermogravimetric analysis, and chemical methods such as Karl Fischer volumetric and coulometric titration. Coulometric Karl Fischer (KF) titration is probably the most commonly applied method [4] as the equipment is fairly inexpensive and the method is easily performed, although where samples are poorly soluble in the methanolic reagents (even when pulverized to aid solubilization), modifications to the protocols have been devised [5]. It is known that some compounds, in particular ketones and aldehydes, also can be problematic [6] with the quantity of water present being over-estimated due to reaction between the methanol solvent with the carbonyl groups.

The National Institute for Biological Standards \& Control (NIBSC) produces a range of lyophilized biological reference materials on behalf of the World Health Organization from a wide variety of sources and molecular types (www.nibsc.org). We have wide experience of testing these materials by coulometric Karl Fischer and although a low moisture environment is required [6], where the containers of such materials have to be opened before addition to the KF cell, and adequate solubility and dispersion of the samples are important, we found the method is robust and straight forward for most biological samples [7].

However, recently we have encountered difficulties with three different biological materials and so have applied an alternative moisture determination method, namely thermogravimetric analysis (TGA). TGA has been reported as a suitable method for moisture determination [8] but because of the risk of thermal decomposition at temperatures above $100^{\circ} \mathrm{C}$ it is combined with evolved gas analysis. This approach has been applied to several specific biological materials before [9] but we have used it on these recalcitrant materials in order to supply QC information to support their release and use.

\section{Materials and Methods}

\subsection{Lyophilized Samples}

Lyophilized biological reference materials were prepared [10] in batches of heat-fused glass ampoules, typically dispensed as aqueous solutions in 0.5 or $1.0 \mathrm{~g}$ aliquots and dried using freeze drying cycles proven to deliver low residual water content (typically around $1 \% \mathrm{w} / \mathrm{w}$ ).

\subsection{Processing the Hygroscopic Samples}

Sample ampoules were opened in a low relative humidity environment using a dry box (Plas Labs, Lansing, MI, USA, supplied by A1-Envirosciences, Cramlington, UK) with moisture maintained below 300 ppm, or a glove bag (CaptairPyramid, Cole Parmer, London, UK) flushed with dry nitrogen to a RH level of 5\% - 10\%.

\subsection{Coulometric Karl Fischer Titration}

Coulometric Karl Fischer titrations were performed on a Mitsubishi CA-100 coulometer, using Aquamicron AX anolyte and CXU catholyte reagents, with a diaphragm-containing cathode (A-1 Envirosciences, Cramlington, UK) which was set up inside the Plas Labs dry box. Samples were opened in a dry environment as described and dissolved in $1-3 \mathrm{ml}$ of the cell anolyte solution, before re-injection back into the Karl Fischer cell. The system was calibrated before use with a commercial water standard solution (Aquamicron P, $4 \mathrm{mg} / \mathrm{g}$ water content, A-1 Envirosciences) and titrations deemed valid only if a $50 \mu \mathrm{L}$ injection gave a water result of $180-210 \mu \mathrm{g}$ with a $\mathrm{CV}$ of $<5 \%$ over three consecutive titrations.

\subsection{Thermogravimetric Analysis}

Thermogravimetric analysis was performed on a Perkin Elmer Pyris 1 system (PETA Solutions, Seer Green, Bucks, UK) fitted with an Accupik autosampler. Known weights of lyophilized samples (2 - $3 \mathrm{mg}$ of crumbled lyophilized powder, packed in with a spatula) were panned in a dry glove bag into hermetically sealed preweighed pans (Perkin Elmer N537 0464) and crimped and re-weighed on the Pyris system to determine sample weight. Samples pans were punctured by the Accupik just before loading onto the TGA balance. Multiple samples were panned for each product and samples of sodium tartarate hydrate of known water content were analysed at the beginning and end of each set as a calibrant.

Gas analysis was performed on a Hiden HRC-20 quadrapole mass spectrometer using helium as the sparge gas. Data was analysed in Pyris and Hiden software and the weight loss related to the water content determined as that weight which corresponded to a peak in the water mass spectra without a corresponding peak in carbon 
dioxide profile.

\subsection{Vapouriser Karl Fisher Titration}

Vapouriser Karl Fischer titration was performed using a VA-100 oven and CA-100 coulometer (A1-Envirosciences) housed within a dry box with a purge gas of dry nitrogen driving the moisture released from the solid sample (placed inside the oven) through the coulometer KF cell. A heating rate of $210^{\circ} \mathrm{C}$ was used with nitrogen flow of $250 \mathrm{ml} / \mathrm{min}$. A standard of dry sodium tartarate of known moisture (LO6027, Mitsubishi, supplied through A1-Envirosciences) was used to check the performance of the vapouriser.

\subsection{Automated Coulometric Karl Fischer Titration}

An automated coulometric Karl Fischer system was set up based upon the Aquafast system (A1-Envirosciences). Samples of lyophilized material were dispensed into septum-sealed autosampler vials in a dry bag environment and then reconstituted with a known aliquot of anolyte. Following a period of time to allow insoluble material to settle an aliquot of the supernatant was titrated into the coulometer KF cell. The coulometer was calibrated using the Aquamicron P solution as for manual coulometric titration.

\section{Results}

\section{Problems with Coulometric Titration}

Three successfully lyophilized materials provided difficulties for us to analyse by coulometric Karl Fischer titration.

A prepared fibrinogen concentrate was difficult to analyse by coulometric Karl Fischer analysis as it had a high dry protein mass ( $45 \mathrm{mg} / \mathrm{g}$ filled solution) and was so compact after lyophilization that it was not possible to completely disperse/dissolve it in anolyte to allow all of the moisture content to be measured. Although KF titration was otherwise normal the residue of non-dissolved/dispersed material meant that there was low confidence that all of the true residual water had been extracted. Determination by TGA analysis was comparatively straightforward (see Figure 1) though only the first mass loss event was due to the removal of bound water (no carbon dioxide evolved). Similar problems were encountered with the coulometric analysis of some lyophilized

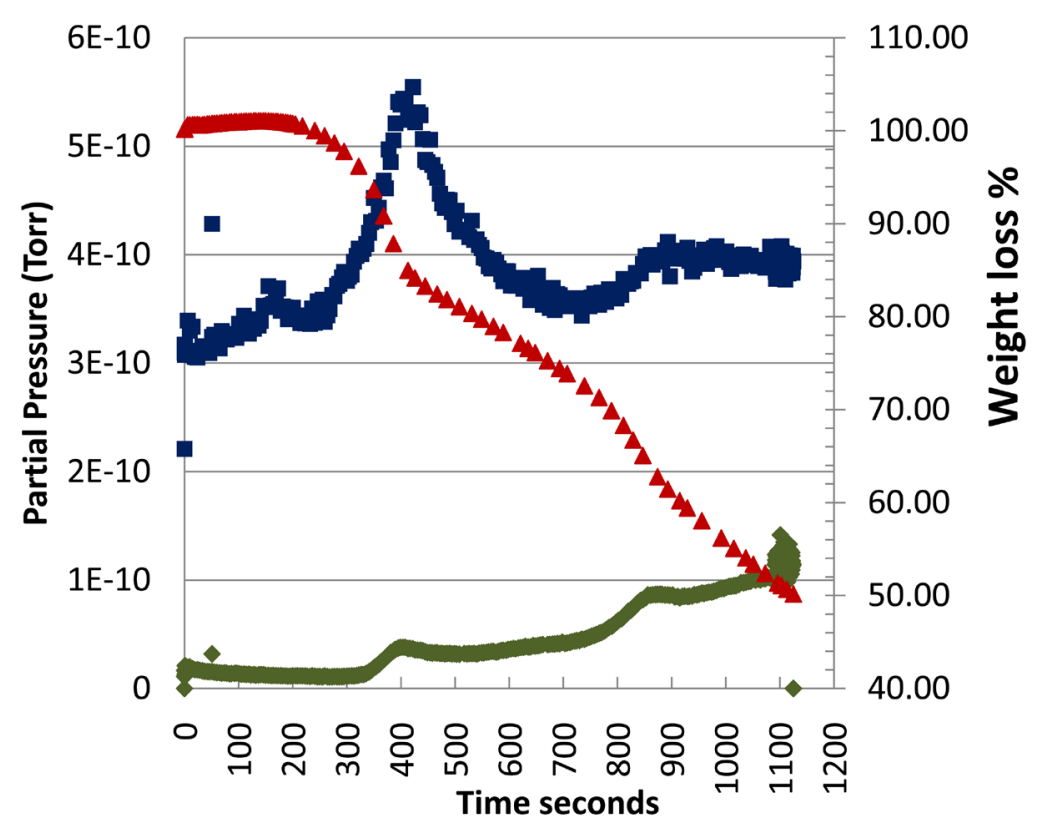

Figure 1. TGA profile of lyophilized fibrinogen concentrate with heating rate of $20^{\circ} \mathrm{C} /$ minute showing three mass loss events (red line), only the first of which (up to 250 seconds) had no associated rise in carbon dioxide (green line) in the EGA profile although there was water (blue line) evolved. 
immunoglobulin preparations (again high protein content at $100 \mathrm{mg} / \mathrm{ml}$ prior to lyophilization) where again the TGA-derived moisture content was much more easily determined but the sample was poorly soluble in the coulometric process. We have previously reported [7] that dense freeze dried cakes of high protein content gained moisture only slowly when exposed to atmospheric air and so the risk of artificially increasing the water content during manipulation of the material to fill the TGA pans was low. A comparison of the moisture content by the two methods is given in Table 1.

A batch of purified heparin presented a different problem for coulometric Karl Fischer titration; although of low dry weight (samples of 2 - 5 mg per ampoule) and dissolving readily, the residual current did not return to the background level within a reasonable titration time ( $<2$ minutes). This problem became worse as repeat samples were titrated, again resulting in the need to regularly change the anolyte and catholyte.

Analysis of the same material by TGA/EGA was straightforward, although the dry weight of the lyophilized material was low and so the total material which could be contained within each pan was limited. Table 2 shows the comparison of the results. Although the w/w moisture content measured by TGA was much higher (3.5\% compared to $0.8 \%$ for coulometric KF) the sample to sample variation in the TGA method was less, though still considerable at $\mathrm{CV}=31 \%$, and the overall moisture content was more comparable to that determined using a vaporiser oven Karl Fischer method (1.7\%). The high CV for all three assays of this material may reflect challenges with handling the material, which has a low dry mass and friable appearance.

A third material which has posed a problem to analyse by coulometric Karl Fischer was a sample of Haemophilus influenzae b (Hib) complex polysaccharide. Within analyzing the first few samples by coulometric KF a white fibrous opaque precipitate built up on the cathode electrode of the Karl Fischer cell (Figure 2) and this prevented the coulometer from regaining the background level of current and so further samples could not be analysed without the cell being emptied, the electrodes cleaned and fresh reagents added.

In comparison the TGA/EGA method was performed without any problem, the only limitation being that the small pan available could only contain a limited volume and as the lyophilized oligosaccharide cake is very light and friable it was difficult to pan more that 1 - $2 \mathrm{mg}$ of powder per sample a typical profile is shown in Figure 3. A lower but broadly comparable moisture content was determined using an automated coulometric Karl Fischer method where fouling of the KF cell was avoided by injecting only part of the reconstituted sample (Table 3).

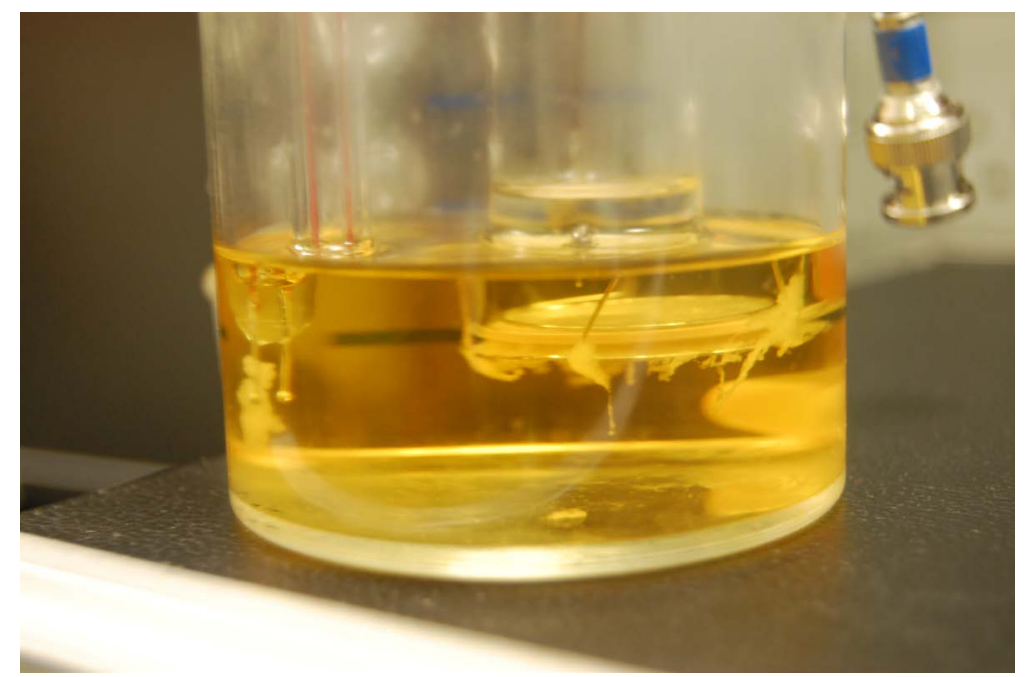

Figure 2. Photograph of KF cell cathode after analysis of Haemophilus influenzae b showing accumulation of opaque fibrous precipitate.

Table 1. Residual moisture content of fibrinogen concentrate by coulometric KF and TGA.

\begin{tabular}{lcc}
\hline & \multicolumn{1}{c}{ Residual moisture content (\% w/w) with number of samples tested and \% CV } \\
\cline { 2 - 3 } & Coulometric Karl Fischer Result & TGA/EGA Result \\
\hline Fibrinogenc oncentrate & $1.02 \%, \mathrm{n}=6, \mathrm{CV}=19 \%$ & $1.22 \%, \mathrm{n}=6, \mathrm{CV}=18 \%$ \\
\hline
\end{tabular}




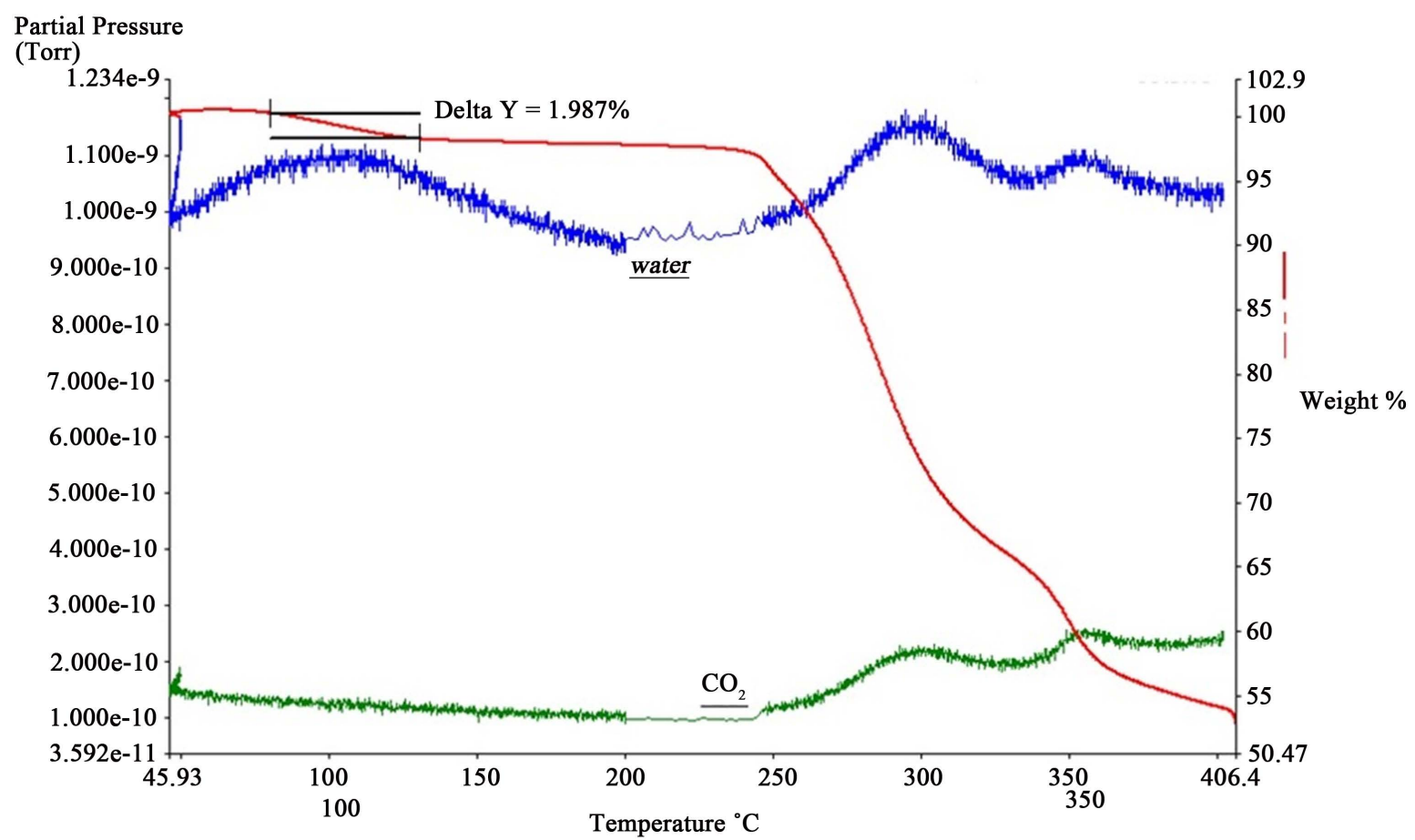

Figure 3. TGA/EGA profile of Haemophilus influenzae b polysaccharide against temperature showing water (blue line) only evolved with the first peak (below $130^{\circ} \mathrm{C}$ ) but carbon dioxide (green line) with subsequent weights losses (red line), indicative of thermal decomposition.

Table 2. Residual moisture content of lyophilized heparin by coulometric KF and TGA.

\begin{tabular}{cccc}
\hline & \multicolumn{3}{c}{ Residual moisture content $(\% \mathrm{w} / \mathrm{w})$ with number of samples tested and \% CV } \\
\cline { 2 - 4 } & Coulometric Karl Fischer Result & TGA/EGA Result & Vaporiser Karl Fischer Result \\
\hline Heparin & $0.78 \%, \mathrm{n}=19, \mathrm{CV}=59 \%$ & $3.5 \% \mathrm{n}=6, \mathrm{CV}=31 \%$ & $1.73 \% \mathrm{n}=3, \mathrm{CV}=50.4 \%$ \\
\hline
\end{tabular}

Table 3. Residual moisture content of lyophilized Haemophilus influenzae b polysaccharide by coulometric KF and TGA.

\begin{tabular}{|c|c|c|c|}
\hline & \multicolumn{3}{|c|}{ Residual moisture content (\% w/w) with number of samples tested and \% CV } \\
\hline & Coulometric Karl Fischer Result & TGA/EGA Result & Automated Karl Fischer Result \\
\hline $\begin{array}{l}\text { Haemophilus influenzae } \\
\text { b polysaccharide }\end{array}$ & $1.45 \%, \mathrm{n}=12, \mathrm{CV}=45 \%$ & $2.04 \%, n=4, C V=8.4 \%$ & $1.14 \% \mathrm{n}=4 \mathrm{CV}=20 \%$ \\
\hline
\end{tabular}

\section{Discussion}

A comparison has been made between TGA and coulometric Karl Fischer for three materials which have presented problems for conventional coulomeric Karl Fischer titration in our hands. The TGA method was capable of analyzing these samples without any fundamental difficulties, other than the need to pack sufficient material into the TGA sample pan. By contrast these samples could not be successfully analysed by coulometric Karl Fischer without costly repeated changing of the reagents and cleaning of the cell every 2 - 3 samples.

The fibrinogen sample presented a well understood issue for coulometric Karl Fischer in that the dry weight protein content was very high and so solubility in methanolic reagent would always be a problem. Replacing some of the reagent with formamide [11] has been advocated in such situations but was not considered suitable in our case due to the toxicity of the reagent. TGA however could readily be used to analyse such materials and because it measures moisture directly in terms of weight loss, it is not affected by chemical cross reactions.

An added complication with understanding the incompatibilities encountered with the heparin (with over long titration times) and Hib polysaccharide (with precipitation of presumably the sample on the cathode) was that 
other batches of similar heparin and Hib polysaccharide materials were readily analysed by coulometric Karl Fischer without these problems being encountered. The apparent precipitation of the Hib polysaccharide on the cathode but not the anode would suggest that this is to some degree a charge-related effect. So as no apparent differences were present from the certificate of analysis disclosed by the suppliers for these batches and those which could be analysed by coulometric KF without an issue, it can only be concluded that it is some unspecified excipient that has caused the effect or changes to the active material which has resulted from some detail of their processing or preparation of which we are not aware. Use of an automated coulometric KF method also permitted analysis of the problematic Hib sample without any visible precipitate forming in the KF cell.

TGA/EGA was able to deal with all of these materials however without problem and so would be a better and more cost-effective method to use for materials of this type in the future. We have in the past used the TGAEGA method principally as a research/troubleshooting option as it is more difficult to set up and expensive in terms of the pans used. Optimising the TGA/EGA system and maintaining it in calibration are time-consuming activities and some of the lessons we have learned, including optimization of carrier helium gas flow rate and pre-equilibrating the sample before beginning a thermal ramp, have been reported previously [7]. One problem which as a user is not easy to address is the limitation of the pan size (fillable volume being around $0.04 \mathrm{~cm}^{3}$ ). Biological materials such as those we often encounter have low dry mass (typically $1-10 \mathrm{mg} / \mathrm{g}$ filled material) and so a large volume pan would enable more material to be loaded per analysis. However, the only larger pans we are aware of are made of steel and would not be punctured by the Accupik needle.

Residual moisture determination is a key quality control test for biopharmaceutical manufacturers and we would recommend thermogravimetric analysis with mass spectrometric gas analysis as an option to consider, especially if problems are experienced with conventional coulometric Karl Fischer titration.

\section{Acknowledgements}

We thank Drs Tim Mann and Paul Gabbott of PETA for help setting up the TGA system and Mr Paul Taylor (A1-Envirosciences) for help with the automated KF system.

\section{References}

[1] Manning, M.C., Chou, D.K., Murphy, B.M., Payne, R.W. and Katayama, D.S. (2010) Stability of Protein Pharmaceuticals: An Update. Pharmaceutical Research, 27, 544-575. http://dx.doi.org/10.1007/s11095-009-0045-6

[2] Towns, J.K. (1995) Moisture Content in Proteins: Its Effects and Measurement. Journal of Chromatography A, 705, 115-127. http://dx.doi.org/10.1016/0021-9673(94)01257-F

[3] Breen, E.D., Curley, J.G., Overcashier, D.E., Hsu, C.C. and Shire, S.J. (2001) Effect of Moisture and Stability a Lyophilized Humanized Monoclonal Antibody Formulation. Pharmaceutical Research, 18, 1345-1353. http://dx.doi.org/10.1023/A:1013054431517

[4] European Pharmacopoeia 8.0 (2014) Chapter 2.5.32 Water: Micro Determination.

[5] Scholz, E. (1984) Karl Fischer Titration. Springer Verlag, Berlin.

[6] May, J.C. (2010) Regulatory Control of Freeze-Dried Products: Importance and Evaluation of Residual Moisture. In: Rey, L. and May, J.C., Eds., Lyophilization/Freeze Drying of Pharmaceutical and Biological Products, 3rd Edition, Informa Inc., New York, 288-316.

[7] Malik, K.P., Duru, C., Ahmed, M. and Matejtschuk, P. (2010) Analytical Options for the Measurement of Residual Moisture Content in Lyophilized Biological Materials. American Pharmaceutical Review, 13, 42-47.

[8] May, J.C., Grim, E., Wheeler, R.M. and West, J. (1982) Determination of Residual Moisture in Freeze Dried Viral Vaccines: Karl Fischer, Gravimetric and Thermogravimetric Methodologies. Journal of Biological Standardization, 10, 249-259. http://dx.doi.org/10.1016/S0092-1157(82)80026-7

[9] May, J.C., Del Grosso, A., Etz, N., Wheeler, R. and Rey, L. (2006) Thermogravimetry and Vapour Pressure Moisture-Applications to Determination of Residual Moisture in BCG Vaccines. Journal of Thermal Analysis and Calorimetry, 83, 31-33. http://dx.doi.org/10.1007/s10973-005-7052-6

[10] WHO (2006) Recommendations for the Preparation, Characterization and Establishment of International and Other Biological Reference Standards (Revised 2004). WHO Technical Report Series 932, Annex 2, 73-131.

[11] Schmitt, K. and Isengard, H.D. (1997) Method for Avoiding the Interference of Formamide with the Karl Fischer Titration. Fresenius' Journal of Analytical Chemistry, 357, 806-811. http://dx.doi.org/10.1007/s002160050253 